\title{
Regional Collaboration Among Countries in the Indian Ocean for Better Coastal and Ocean Governance
}

\author{
Steen Christensen, Raquibul Amin, \\ Ghulam Qadir Shah
}

\begin{abstract}
The Mangroves for the Future initiative (MFF) has operated since 2006 as a strategic regional and partnership-led programme working in response to the continued degradation of coastal ecosystems, which threatens the livelihoods and security of coastal communities and makes them more vulnerable to impacts from climate change. MFF follows the principle that healthy coastal ecosystems (principally mangroves, coral reefs and sea grass beds) can contribute significantly to human well-being and the resilience of man and nature to climate change.
\end{abstract}

In its initial phase to 2010, MFF supported India, Indonesia, Maldives, Seychelles, Sri Lanka and Thailand to deliver a targeted response to the Indian Ocean tsunami in 2004. Since then, country membership has expanded to include Bangladesh, Cambodia, Myanmar, Pakistan and Viet Nam and the initiative has grown towards a wider response to the current and emerging challenges for coastal management in the Indian Ocean and South China Sea regions. MFF is also engaged with Malaysia on an outreach basis; and with the Philippines as a dialogue country.

Co-chaired by International Union for Conservation ofnature (IUCN) and United Nations Development Programme (UNDP) and with ASEANCentre for Biodiversity (ACB), Food and Agricultural Organization of the United Nations (FAO), United Nations Environmental Programme (UNEP), and Wetland International (WI) as institutional partners, MFF provides a unique platform for representatives from governments, civil society and private sectors to meet, discuss and take actions together at both regio nal and national scales. At the regional level, MFF is governed by a Regional Steering Committee that provides strategic leadership for policy change and advocacy and also serves as a forum for country to country information exchange and discussions on emerging issues of regional importance. Within each MFF member country, a National Coordination body has oversight on the programme implementation as guided by its 
National Strategy and Action Plan and delivery of the small, medium and regional grantfacilities ${ }^{1}$.

Through its inclusive partnership approach, MFF has significantly strengthened the collaboration between the key stakeholders from governments, civil society, and private sectors addressing national and regional coastal zone management issues. In particular, the MFF initiative has supported national and local governments in developing and implementing participatory and sustainable resource management strategies, and, through awareness and capacity building activities, empowered coastal communities to participate in the decision making processes relating to the management of the natural resources on which they depend for their livelihoods.

The present paper elaborates successful examples of regional collaboration initiated through MFF that have contributed to better coastal governance in India Ocean region. The examples include the transboundary cooperation between Pakistan and Iran in Gawatar Bay, between India and Sri Lanka for in the Gulf of Mannar, and between Guangxi and Quang Ninh Provinces in China and Viet Nam. The paperalso highlights the importance of the Sustainable Development Goals, especially the Goal 14 and concepts like Blue Economy for sustainable ocean and coastal management in the light of the rapid development in maritime trade, race for exploration of the ocean bed for mineral resources, unsustainable exploitation offish resources, and increasing pollution load to ocean waters from rapidly developing coastal urban growth in the Indian Ocean region.

Keywords: Environment Conservation, Marine Pollution, Mangroves, Regional Collaboration, Indian Ocean.

1 "Greater maritime link urged among nations in the Indian Ocean", The Express Tribune, February 13, 2017, https://tribune.com.pk/story/1324937/greatermaritime-link-urged-among-nations-indian-ocean/ 


\section{Introduction}

Mangroves for the Future (MFF) was established in 2006 as a partnership-based initiative to help the six countries most devastated by the 2004 tsunami (India, Indonesia, Maldives, Seychelles, Sri Lanka and Thailand). Following extensive consultations, MFF was designed as a collaborative platform for multiple stakeholders to work together nationally and regionally to promote investment in coastal ecosystems as a form of natural infrastructure to provide physical protection against natural hazards and support the livelihood needs of ecosystem dependent coastal communities. Based on 15 thematic programmes of work, grant projects were implemented through local organizations (mainly NGOs and CBOs) in each country as the main means of providing this support 2 .

Mangrove forests once covered more than $200,000 \mathrm{~km}^{2}$ of protected tropical and subtropical coastlines ${ }^{3}$. They are vanishing worldwide from 1 to $2 \%$ per year, a rate greater than or equal to drops in neighbouring coral reefs or tropical rainforests $(4,5,6,7)$. Losses are happening in almost

2 "Mangroves for the future holds 14th regional steering committee meeting", The Global New Light of Myanmar, September 30, 2017, http://www.globalnewlightofmyanmar.com/mangroves-future-holds-14thregional-steering-committee-meeting/

${ }^{3}$ Daphne J. Osborne, "SPALDING, M. D., BLASCO, F. \& FIELD, C.D. (Eds) 1997. World Mangrove Atlas. International Society for Mangrove Ecosystems, Journal of Tropical Ecology 14, no. 5 (1998): 723-24. doi:10.1017/S0266467498300528.

4 "STATUS AND TRENDS IN MANGROVE AREA EXTENT WORLDWIDE", Fao.Org, 2021, http://www.fao.org/3/j1533e/J1533E00.htm.

5 Daniel M. Alongi, "Present State And Future Of The World's Mangrove Forests", Environmental Conservation 29, no. 3 (2002): 331-349.

6 Valiela, I., Bowen, J.L. \& York, J.K. (2001). Mangrove Forests: One of the World's Threatened Major Tropical Environments: At least 35\% of the area of mangrove forests has been lost in the past two decades, losses that exceed those for tropical rain forests and coral reefs, two other well-known threatened environments.

Bioscience, $51(10)$, 807-815.

7 Stone, R. (2007). A world without corals? Science, 316(5825), 678-681. 
every country that has mangroves, and rates continue to rise more quickly in developing countries, where $>90 \%$ of the world's mangroves are situated. The accuracy and detail of the UN Food and Agriculture Organization data on which these observations are based may be debatable, but mangrove losses during the last quarter century range steadily between 35 and $86 \%$. As mangrove areas are becoming smaller or patchy, their long-term existence is at great risk, and vital ecosystem services may be lost. Where mangrove forests are cleaned for aquaculture, urbanization, or coastal landfill or decline due to indirect effects of pollution and upstream land use, their species productivity is expected to decline rashly, because the number of mangrove plant species is directly connected with forest size ${ }^{8,9}$. Examples from other ecosystems have shown that species extinctions can be followed by a loss in functional diversity, particularly in species-poor systems like mangroves, which have low redundancy per $\mathrm{se}^{10}$. Therefore, any further deterioration in mangrove area is likely to be followed by augmented functional losses. Mangroves are already critically endangered or upcoming extinction in 26 out of the 120 countries having mangroves ${ }^{11}$.

In the second phase (2010 - 2014), Bangladesh, Pakistan and Vietnam became full member countries and the MFF programme evolved to better meet emerging needs and priorities - climate change, gender equality and effective communications were introduced as important cross-cutting themes in all MFF interventions. MFF supported more than 175 small to larger projects, which necessitated the development of a comprehensive Knowledge Management and Communications (KMC) strategy at regional level. Results and lessons

8 Norman Duke, Marilyn Ball, and Joanna Ellison, "Factors influencing biodiversity and distributional gradients in mangroves." Global Ecology \& amp; Biogeography Letters banner, 7, no.1,(1998): 27-47.

9 Aaron M. Ellison, (2002). "Macroecology of mangroves: large-scale patterns and processes in tropical coastal forests," Trees Structure \& Functions, 16, no 2-3, (2002): 181-194

10 Owen L. Petchy and Kevin J Gaston. "Extinction and the Loss of Functional Diversity." Proceedings of the Royal Society B: Biological Sciences 269, no. 1501 (August 22 , 2002): 1721-27.

11 Global Marine Species Assessment, Old Dominion University, Norfolk, Virginia, (2018). 
learned from the individual projects were captured via a field level Monitoring, Learning, and Evaluation system and then disseminated at the local, national and regional levels through online media and through seminars, workshops, national and cross country study tours, training courses, etc. in accordance with the MFF KMC strategy. Danida provided dedicated support to MFF from 2012-2015 for mainstream Climate Change Adaptation, with a country focus on Bangladesh, Indonesia and Vietnam. Through strategic project implementation and special studies focusing on the Ecosystem-based Approach and environmental valuation, this support generated good knowledge and experience in best practices for Climate Change Adaptation, including co-management mechanisms and other inclusive benefit- sharing mechanisms. The support also provided an opportunity to develop the MFF Resilience Framework, including a Resilience Assessment Protocol that now applied in all MFF member countries to guide strategic delivery of MFF projects, including project selection, monitoring and reporting in accordance with national policy priorities.

In Phase 3 (2014 - 2018) MFF expanded to 11 member countries with the addition of Cambodia and Myanmar. Based on the experience and lessons learned from the two earlier phases the MFF Resilience Framework is now being applied as a guiding principle for programme implementation with the overall focus on strengthening the resilience of ecosystem-dependent coastal communities applying nature-based solutions.

The main objective of the current MFF programme is to increase the resilience of people living in traditional coastal communities who are most vulnerable to climate change and natural hazards. Restoring and conserving coastal ecosystems, especially mangroves, coral reefs and sea grasses, and sustainably managing the ecosystem services they provide, are regarded as fundamental to achieving this objective. This is mainly an adaptation approach, which applies the principle that healthy coastal ecosystems contribute significantly to human health, well-being and food security across the coastal regions of Asia. This is expressed in the outcome objective of Phase 3, which is: 'Resilience of Ecosystem Dependent Coastal Communities Strengthened.' Delivery of this outcome is through three output objectives and four cross cutting themes: 
Output Objective 1: Knowledge generated, disseminated and applied to sustainable management of coastal ecosystems. Under this objective MFF aims at strengthening the information base for sustainable management by enhancing access to and promoting effective use of knowledge and best practices relating to coastlines and communities vulnerable to climate change and other natural hazards.

Output Objective 2: Key stakeholders empowered to engage in decision-making in support of sustainable management of coastal ecosystems. Considering capacity-building and strategic knowledge dissemination and communication priority programme elements contributing to empowerment, under this objective MFF aims at increasing the awareness and capacity of civil society and private sector to engage in inclusive management of coastal social ecological systems. Sustainable livelihoods and well-being of coastal populations vulnerable to climate change risks and impacts are enhanced through community-managed projects based on the MFF modalities where project selection is guided by a comprehensive resilience assessment.

Output Objective 3: Coastal governance, enhanced to promote integrated and inclusive management. Under this objective MFF aims at strengthening the capacity of local, national and regional governance institutions for integrated coastal management, engaging with key business sectors to promote climate-resilient and sustainable business practices based on blue economy and green growth principles. By applying a soft governance approach and bringing together relevant sectors under the umbrella of the MFF National Coordinating Bodies, MFF has made a significant contribution to overcoming traditional "siloed" approaches and is developing a common understanding of coastal issues and the nature-based solutions which can be applied to address them. 


\section{Cross Cutting Themes}

Where relevant, in addition to the focus areas listed above, projects supported by the MFF Grant Facilities are expected to address four cross-cutting themes: Climate Change, Gender Integration, Conflict Sensitivity and Property Rights and Resource Tenure. These are given special consideration in the MFF Resilience Assessments that provide the baseline information used to support project identification and selection, and the subsequent monitoring of project results against a known baseline.

\section{MFF Governance Structure}

The partnership nature of MFF is reflected in the MFF Governance Structure that was established in the early phases of MFF and is one of the main reasons for the recognition and long term success of MFF. The MFF governance structure in addition to providing oversight for programme implementation is also the main mechanism for policy influence and national as well as regional level.

The highest-level decision-making body of MFF is the Regional Steering Committee (RSC). The RSC is co-chaired by IUCN (Asia Regional Office) and UNDP (Regional Centre in Bangkok) and has representatives from each of the MFF member countries, and a number of institutional partners including ASEAN ACB, FAO, UNEP and Wetlands International. Representatives from the core programme donor countries, Denmark (Danida), Norway (Norad and Royal Norwegian Embassy) and Sweden (Sida) are also invited to RSC meetings as observers. The RSC provides strategic leadership for policy change and advocacy at regional level and also serves as a highly appreciated opportunity for country-to-country information exchange and discussions on emerging issues of regional importance. Depending on availability of funds, the RSC provides country allocations for implementation of the MFF Grant Facilities and also assigns allocations for major regional learning and knowledge sharing activities. The annual RSC meetings are hosted by the member countries on a rotational basis which provides a much-appreciated opportunity for the hosting country to arrange field site visits to showcase ongoing field work. Further, a regional learning event is usually arranged back-to-back with the RSC meetings in consultation with the host country. 
The MFF Management Committee (MC) is a committee under the RSC that meets annually to facilitate decision making between the RSC meetings. The MC is comprised of the two co-chairs plus, selected on a rotational basis, three NCB Chairs as country representatives and one institutional partner representative. The MC established in 2013 and has proved very useful to expedite decisions with regards to implementation and budget reallocations that would otherwise have delayed implementation. By taking over the more technical aspects of project and programme management the RSC has been able to focus more on strategic issues of regional relevance. The MC meetings are held in Bangkok.

Within each MFF member country, a National Coordinating Body (NCB) has oversight of MFF activities at the national level. Here again, reflecting the partnership approach of MFF, NCBs include relevant government departments, civil society organizations including NGOs, academia, and individual experts and, in most countries, private sector representatives. Guided by a National Strategy and Action Plan (NSAP) reflecting national priorities for sustainable coastal development, the NCB meets several times per year to oversee MFF implementation, including the strategic delivery of the grant facilities, at country level. However, the NCBs are particularly recognized and appreciated as multi sectoral, soft governance bodies for informal knowledge exchange and efficient platforms for national policy influence.

In each MFF member country the work of the NCB is assisted by a small national secretariat within the country office of IUCN, UNDP (Maldives) or Wetlands International (Indonesia). A National Coordinator is assigned to the country secretariat office to provide dedicated support to the policy work of the NCB and coordinate with the MFF Regional Secretariat in the IUCN Asia Regional Office in Bangkok.

\section{Project Implementation Modalities}

The MFF Grant Facilities were introduced in 2007 as the main vehicle to deliver on-the-ground results for the well-being of coastal ecosystems and coastal resource-dependent communities. Projects supported by the MFF Grant Facilities are expected to address one or more of the MFF output objectives and the nine strategic focus areas as well as relevant cross cutting issues. 
The strategic delivery of the MFF Grant Facilities is guided by the NSAPs, reflecting national priorities for sustainable coastal development, and comprehensive resilience assessments of the targeted coastal SocioEcological Systems. The MFF Resilience Assessment Protocol (RAP) ${ }^{12}$ has been developed as a participatory, stakeholder led research and planning framework for undertaking baseline assessments of the target areas. The framework provides a basis for designing and implementing priority actions for coastal management, engaging governments (the main duty-bearer) as well as civil society groups (the right holders) in the coastal planning and management processes. The inclusive approach of the RAP allows integration of local as well as scientific 'knowledge' of social and ecological systems and generates a picture of the system dynamics and interactions through a consensus lead process - with representation of all stakeholders' ideas and concerns. In this way the RAP provides a functional analysis of the targeted Social-Ecological Systems (SES) to prioritize actions for immediate investment to enhance social-ecological resilience while, at the same time, also engages the community and ensures that the development process is locally owned. The RAP has also provided a baseline for facilitating the Human Rights Based Approach (HRBA) as an integral component of the MFF development planning approach that addresses a number of cross cutting themes including gender equality, conflict sensitivity, property rights and resource tenure. The RAP has been a mandatory tool for strategic implementation of the MFF grant facilities since the beginning of Phase 3 and, in addition to guiding project selection, also serves as a strong basis for monitoring and evaluation and for result based reporting.

Small Grant Facility (SGF): The main aim of the SGF is to finance small projects (up to a maximum of USD 25K) in order to support strategic and tailor-made local community action for the management of coastal ecosystems and their use on a sustainable basis. Small Grants are meant to support local NGOs, which are often best positioned to work with local communities and which have a good understanding of local ecological and social-institutional conditions.

12 "Mangroves for the Future Twelfth Regional Steering Committee Meeting “ (RSC-12) Progress Report. (2015), https://info.undp.org/docs/pdc/ Documents/MDV/RSC\%2012\%20MV\%20Report\%20CR.pdf 
SGF implementation follows a strategic approach by targeting welldefined geographic and thematic areas prioritized by the MFF National Coordinating Body (NCB) and reflected in the NSAP, with the long-term aim of developing the resilience of ecosystem-dependent coastal communities. As an overarching principle, small projects shall respond to identified needs or issues identified through the resilience assessment and shall remain grounded and linked with the realities and needs of local communities.

In addition to providing direct environmental and livelihood benefits at a local level, SGF projects are expected to offer tangible 'models' to inspire policy-making and they should include concrete measures to ensure a participatory approach, promote gender equality and secure livelihoods for marginalized groups. In this way, SGF projects help in linking experiences and lessons learned at the household and community levels to the dynamics of policy and decision-making about coastal area planning and investment. The SGF also provides as an opportunity for the countries to engage more effectively with the private sector and to attract private sector co-funding.

Medium Grant Facility (MGF): The MGF established in 2010 with the intention to contribute more significantly to strengthening the resilience of ecosystem dependent coastal communities by addressing specific resilience parameters identified through comprehensive site assessments guided by the MFF Resilience Framework. The MFF contribution to medium projects is up to USD 100,000. The Projects under the MGF are expected to build on expanding from successful small grant projects and to examine more complex and often multidisciplinary issues affecting coastal zone management as prioritized by the NCB.

Regional Grant Facility (RGF): The RGF was introduced in 2010 to address trans-boundary coastal zone management issues spanning two or more countries, to consolidate existing knowledge particularly relevant to coastal resources management, and to conduct research on emerging issues to acquire new knowledge. The maximum contribution from MFF to a regional project is USD 200,000. 


\section{Project Outcomes}

The table (1) gives an oversight of the number of projects implemented by MFF to date and clearly reflects that with a limited investment MFF has succeeded in implementing a high number of projects and generated a large amount of co-founding. The high number of small grants has been directed primarily towards national ecosystem restauration and rehabilitation, livelihood diversification, and improving the knowledge base and capacity for better resource management. An oversight of projects and project outcomes can be found at the MFF website: www.mangrovesforthefuture.org

\begin{tabular}{|c|c|c|c|}
\hline Grant facility & $\begin{array}{c}\text { No of } \\
\text { projects }\end{array}$ & $\begin{array}{c}\text { MFF } \\
\text { contribution, } \\
\text { USD }\end{array}$ & $\begin{array}{c}\text { Total } \\
\text { Co-financing, } \\
\text { USD }\end{array}$ \\
\hline Small grants & 313 & $4,140,077$ & $2,798,566$ \\
\hline Medium grants & 8 & 530,894 & 190,576 \\
\hline Large grants & 10 & $2,481,100$ & $1,294,978$ \\
\hline Regional grants & 5 & 988,058 & 492,613 \\
\hline Total grants & 336 & $8,140,129$ & $4,776,733$ \\
\hline
\end{tabular}

Table 1: MFF project implementation 2006 - 2016

MFF grant projects have significantly strengthened the collaboration between the key stakeholders from governments, civil society, and private sectors by addressing national and regional coastal zone management issues. In addition, MFF fosters regional cooperation through its regional grant projects as indicated in the following examples.

Cooperation on Gulf of Mannar between India and Sri Lanka

MFF has facilitated transboundary cooperation between the government of Sri Lanka and India on the issue of fisheries management in the Gulf of Mannar. The rich resources of the Gulf are utilized by the 
coastal communities in Mannar, Kilinochchi and Jaffna Districts in Sri Lanka, and by the fishing communities known as Marakeyars in South India. For almost 30 years, due to the separatist war in Sri Lanka, fieldbased scientific studies have been almost non-existent in the Gulf of Mannar. To address the gap in scientific data, a joint assessment of coral reefs, mangroves and seagrasses in the Gulf of Mannar was undertaken by the two countries, resulting in a joint recommendations paper on the conservation and management which was presented at a high-level meeting of Senior Government Officials of India and Sri Lanka. The event resulted in nine policy recommendations and the formation of a joint technical group to guide the work of a joint Fisheries Commissions.

\section{Sino-Viet Nam initiative}

MFF is supporting a trans-boundary initiative between Quang Ninh in Vietnam and Guangxi in China to develop organic aquaculture and mangrove ecosystem conservation that will eventually improve the livelihood of the local communities, strengthen economic exchanges and promote sustainable fisheries development between Vietnam and China. Dialogues are also on-going to establish modalities for cooperation between scientific institutions and transfer of technologies.

\section{Regional initiatives of specific interest to Pakistan}

Pakistan has been a partner of three regional initiatives supported by MFF as follows:

Pakistan-Iran Initiativ: Gwatar Bay is a trans-boundary bay situated on the coast of the Sistan-Balochistan province of Iran and the Balochistan province of Pakistan. It is one of the largest embayment along the entire coast of the two countries and covers an area of around $1,000 \mathrm{~km} 2$ (including the adjacent floodplain) and $64 \mathrm{~km}$ of coast. The bay is fed by the largest rivers of Balochistan: the Dashtriver in Pakistan and the Bahu-Kalat river in Iran. The uniqueness of this area led to the designation of two Ramsar sites: the Govater Bay and Hur-e-Bahu Ramsar site in Iran and the Jiwani Coastal Wetlands Ramsar site in Pakistan. These two sites form an almost unique entity, a transboundary coastal ecosystem protected under the Ramsar Convention. A significant coastal portion of the bay in Iran is included in the Bahu Kalat Protected Area, also an Important Bird Area (IBA). The Eastern part of the Gwatar Bay has been proposed as an Ecologically or Biologically Significant 
Marine Area (EBSA), due to its importance of marine turtle reproduction.

Despite the recognition of the international importance of the bay of the two countries by declaring two Ramsar sites, the ecosystem of the Gwatar Bay still lacks a conservation or management plan. The area is also under threat from over-fishing, pollution and climate change. Through NCB Pakistan, IUCN Pakistan took the initiative to develop a project proposal to raise funds to put in place a transboundary framework for management of Gwatar Bay. This project provides a platform for dialogue, coordination, and cooperation between Iran and Pakistan on the conservation of the Gwatar Bay area and supports the development and implementation of a joint strategy and plan for its management. The project is also intended to support research and dialogues with key stakeholders to provide a sound foundation for the sustainable management of the resources of the Bay for the long-term benefits of coastal communities in both countries.

Income for Coastal Communities for Mangrove Protection: The project 'Income for Coastal Communities for Mangrove Protection' aimed to develop a low-cost mechanism enabling investors to responsibly promote mangrove conservation and restoration, carbon emissions reduction and sustainable development through the provision of funding to local communities. The project was jointly implemented in Pakistan, Thailand and Viet Nam and has resulted in the design of new low-cost mechanisms to ensure mangrove areas are monitored and protected, carbon stocks are assessed, and local communities are involved in and benefit from project activities ${ }^{13,14,15}$.

13 Julien Rochette, Sebastian Unger, Dorothee Herr., David Johnson., Takehiro Nakamura, Tim Packeiser, Alexander Proelss, Martin Visbeck, Andrew Wright, and Andrew Cebrain, "The regional approach to the conservation and sustainable use of marine biodiversity in areas beyond national jurisdiction", Marine Policy, 49, (2014): 109-117.

14 Adalberto Vallega, "The regional approach to the ocean, the ocean regions, and ocean regionalization-a postmodern dilemma", Ocean \& Coastal Management, 45(2002): 721-760.

15 Quantin A. Hanich and Yoshitaka Ota, (2013). "Moving beyond rights-based management: a transparent approach to distributing the conservation burden 
Resilience to Sea Level Rise : The regional initiative 'Resilience to sea level rise induced coastal erosion' was implemented jointly by Pakistan and Thailand, with the objective to develop a regional strategic approach to understand vulnerability to coastal erosion and implement pilot projects to test and refine priority mitigating interventions. Country assessments and capacity building workshops guided reviews and analysis of coastal erosion status and relevant policies, legislation, and institutional structures. The increased understanding of the coastal erosion situation and policies in Pakistan and Thailand led to the development of coastal erosion management plans and implementation of priority pilot interventions. In Pakistan, the results from the regional initiative were included in the Pakistan National Assessment Report on Coastal Erosion developed by MFF Pakistan. The report was presented to the Senate Standing Committee which subsequently directed the relevant authorities to follow its recommendations.

\section{Conclusion}

The Indian Ocean is the world's preeminent seaway for trade and commerce. It is also endowed with a wealth of natural resources, which are largely untapped. However, as the economy of the countries around the Indian Ocean rim is rapidly rising, the race for exploiting the rich ocean resources are also on the rise. Oceans are seen as the next frontier for economic development. However, the world's oceans, including the Indian Ocean are already under pressure from overexploitation, illegal fishing, land-based pollution and climate change, all of which are caused primarily by human actions. Degradation of ocean health is a security threat as well as it can create global socioeconomic problems, including health, safety and financial risks.

The Sustainable Development Goals (SDGs), and specifically SDG 14 aims to sustainably manage and protect marine and coastal ecosystems from pollution, address impacts of ocean acidification, enhance conservation and the sustainable use of ocean-based resources through international law. Development of a strategic outlook for Indian Ocean for 2030 as in the theme of the workshop needs full adoption of SDG 14-"conserve and sustainably use the oceans, seas and marine resources for sustainable

and benefit in tuna fisheries," The International Journal of Marine \& Coastal Law, 28, (2013): 135-170. 
development"- and its targets agreed upon by the UN member states to help guide decision making with regards to oceans.

The Sustainable Development Goals and in particular Goal 14 are connected to MFF's Strategic focus areas: coastal rehabilitation, livelihood support, resilience-building and empowerment; with climate change and gender as key cross-cutting issues. Through its regional partnership based approach the MFF initiative provides a platform for its member countries to strategically pilot innovative solutions to strengthen integrated coastal management.

The MFF partnership based approach, and in particular its governance structure has proven to be one the main factors contributing to the long term success of MFF to deliver national results with a regional impact. The MFF partnership principle ensures representation of government, civil society and private sector in both national and regional programme management. Applying a soft governance approach, the MFF Regional Steering Committee and National Coordinating Bodies function as unique and efficient platforms for policy influence at the national and regional level while at the same time ensures regional and national relevance of the programme. 